\title{
Analysis of ITS1 sequences and genetic relationships between populations of ridgetail white prawn, Exopalaemon carinicauda, in the East China Sea
}

\author{
X.Z. Zhang ${ }^{1}$, X.Q. Cheng', Y.X. Yu', H. Shen ${ }^{2}$ and X.H. Wan ${ }^{2}$ \\ ${ }^{1}$ School of Life Sciences, Nantong University, Nantong, Jiangsu, China \\ ${ }^{2}$ Institute of Oceanology and Marine Fisheries of Jiangsu, Nantong, Jiangsu, China \\ Corresponding author: X.H. Wan \\ E-mail: zxzlyg@aliyun.com \\ Genet. Mol. Res. 14 (4): 12316-12322 (2015) \\ Received May 6, 2015 \\ Accepted July 23, 2015 \\ Published October 9, 2015 \\ DOI http://dx.doi.org/10.4238/2015.October.9.20
}

ABSTRACT. Internal transcribed spacer 1 (ITS1) sequences from wild-type Exopalaemon carinicauda $(\mathrm{N}=124)$ from the East China Sea were amplified and sequenced. Sequences were polymorphic and ranged from 388 to 583 bp in length. The average content of GC in sequences was significantly higher than that of AT. Altogether, 604 mutant sites with 123 haplotypes were detected; $46.7 \%$ were polymorphic sites. The genetic diversity index of population $\mathrm{Y}$ was highest, and the lowest was population X. Eight microsatellite sequences were detected; the most-repeated sequences were (GA)n, (AG)n, (GT)n, (TG)n, (TC)n, and (CT)n. Analysis of molecular variance revealed that genetic differentiation among the four populations were very weak, or modest. A molecular evolutionary tree was constructed using the neighbor-joining method and MEGA 6.0, and the phyletic evolutionary relationships among several Palaemonidae species examined. The phylogenetic tree showed that individuals of the same species, as well as the species of the same genus, clustered together, consistent with morphological classifications.

Key words: Exopalaemon carinicauda; Internal transcribed spacer 1; Genetic diversity; Phylogenetic analysis 


\section{INTRODUCTION}

Exopalaemon carinicauda (Crustacea: Decapoda: Natantia: Palaemonidae: Exopalaemoni) is widely distributed in the East China Sea (Li et al., 2012). This medium-size economic prawn is aeurythermic, euryhaline, heterophagous, and has a high reproductive rate. Recent structural changes to marine fishery resources have led to the recession of natural resources; the rapid increase of commercial values; and the expansion of artificial culture. Research on provenance resources and genetic diversity of $E$. carinicauda will facilitate the healthy and sustainable development of this important economic prawn.

The ribosomal RNA (rRNA) genes of eucaryotes are stored as tandem repetition in the nucleolus; 18S, 5.8S, and 28S constitute a transcriptional unit (Zhao et al., 2012; Monard et al., 2013). The first transcription spacer in ribosomal DNA is a non-coding spacer between $18 S$ and $5.8 S$, while the second transcription spacer is a non-coding spacer between $5.8 S$ and $28 S$. The Internal transcribed spacer (ITS) zone (including ITS1 and ITS2) of the nuclear genome feature slow selection pressure and rapid evolution, due to the absence of mature ribosomes. Moreover, rich genetic information can be acquired from a relatively short sequence. Thus, the ITS zone gradually becomes an excellent molecular genetic marker for the study of genetic relations, phylogenetic relations, and genetic diversity among close populations (Bazzicalupo et al., 2013; Estrada-Bárcenas et al., 2014). For these purposes, the modestly conserved sequence ITS1 is widely used (Fong al., 2013; Wang et al., 2014).

Until now, research on $E$. carinicauda has focused mainly on biological characteristics, living habits, culture and breeding technology, reproductive biological habits, and disease prevention and control (Xu et al., 2010; Duan et al., 2013; Zhang et al., 2014a,b), but rarely on molecular genetics. Genetic diversity from the perspective of ITS1 has not been reported for this species. In this study, we analyzed the ITS1 sequences from 124 E. carinicauda individuals from four geographical populations, and studied genetic diversity among the populations. The data presented here will provide a scientific basis for protection and utilization of resources of the $E$. carinicauda prawn.

\section{MATERIAL AND METHODS}

Wild type E. carinicauda were collected from XiangShan, NanTong, YanCheng, and RiZhao in the East China Sea. The four populations were marked as populations $\mathrm{X}, \mathrm{N}, \mathrm{Y}$, and R, respectively (Figure 1).

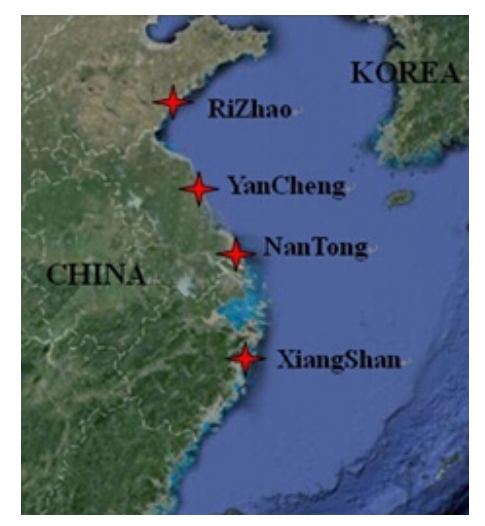

Figure 1. Exopalaemon carinicauda sample collection sites. 
Between 30 and 32 individuals were randomly selected per population, and approximately $100 \mathrm{mg}$ abdominal muscles collected from each. Tissues were ground for DNA extraction using an animal genomic DNA extraction kit (Sangon, Shanghai, China). The concentration and purity of DNA were measured with a DNA quantitative instrument and analyzed by electrophoresis on a $1 \%$ agarose $(\mathrm{w} / \mathrm{v})$ gel. Samples were stored at $-20^{\circ} \mathrm{C}$ until further analysis.

Primers were designed by Oligo 6.0, based on sequence of ITS1 (GQ369794.1) in GenBank, and were: 5'-TAACAAGGTTTCCGTAGGTG-3' (ITSF1), and 5'-AGCGAT CCACCATTAGAAGT-3' (ITSR2). Conditions for PCR were: 5 min denaturation at $95^{\circ} \mathrm{C} ; 50$ cycles of $45 \mathrm{~s}$ at $94^{\circ} \mathrm{C}, 45 \mathrm{~s}$ at $56^{\circ} \mathrm{C}$, and $50 \mathrm{~s}$ at $72^{\circ} \mathrm{C}$; and a final step of $7 \mathrm{~min}$ at $72^{\circ} \mathrm{C}$. Amplification products were detected on a $1.0 \%$ agarose $(\mathrm{w} / \mathrm{v})$ gel and sequenced by Sangon (Shanghai).

Detected sequences were compiled on Bioedit V7.2.5, with the help of artificial check, then matched on ClustalX V1.83 and their lengths measured. Haplotypes, haplotype polymorphisms, polymorphic sites, average number of nucleotide differences, and nucleotide diversity index were computed using DnaSp V5.0, and simple repeated sequences were searched on SSRHunter V3.1. The distributions and genetic differentiation coefficient ( $F$-statistics, $\left.F_{\mathrm{ST}}\right)$ of genetic variation between and within populations were estimated by analysis of molecular variance using ARLEQUIN V3.5.1.3. The significance of $F_{\mathrm{ST}}$ was examined by permutation tests (1000 repetitions). Base composition, mutant sites, parsimony informative sites, switch/transversion in sequences, and the between-population Kimura2-paramter genetic distance were computed on MEGA V6.0. A phylogenetic tree was built using the neighbor-joining method; the support value at each node was expressed as the bootstrap value from 1000 replicate tests.

\section{RESULTS}

ITS1 sequences of $E$. carinicauda varied in length but the differentiation amplitude was low. After 18S rRNA and 5.8S rRNA gene sequences at the two sites were deleted, the ITS1 lengths of populations $X, Y, R$, and $N$ were, respectively, 388-422, 409-501, 412-526, and 395-583 bp. Base compositions of ITS1 sequences $(N=124)$ are listed in Table 1. Base contents were not significantly different among the four populations, but GC content was significantly higher than AT content. Altogether, 604 mutant sites were detected; $46.7 \%$ were polymorphic sites. The proportion of switch sites among ITS1 mutant sites was higher than that of transversion sites. Nine ITS1 sequences (GQ369796, EU346851, EU118285, HM804252, HM590579, GQ369795, EU118286, GQ369793, and EU373484) from three additional Palaemonidae species were extracted from GenBank. These sequences were length-variable, ranging from 345 to $1485 \mathrm{bp}$. The GC contents among the four Palaemonidae species were largely different (approximately 40.5 to $63.1 \%$ ). In all species, GC contents were higher than AT contents, with the exception of Macrobrachium nipponense. Microsatellite sequence was composed of 1-6 nucleotides, in the form of tandemrepeated fragments.

Table 1. Nucleotide compositions (\%) of ITS1 from four wild Exopalaemon carinicauda populations.
\begin{tabular}{lccccc}
\hline Population & T & C & A & C+G & Length (bp) \\
\hline X & 20.86 & 24.05 & 20.36 & 58.78 & $388-422$ \\
Y & 21.70 & 23.81 & 20.78 & 57.52 & $409-501$ \\
R & 20.89 & 25.90 & 22.26 & 56.78 & $412-526$ \\
N & 21.41 & 22.90 & 20.09 & 58.50 & $395-583$ \\
\hline
\end{tabular}


Eight microsatellite sequences were detected in the ITS1 sequences; the most-repeated sequences were (GA)n, (AG)n, (GT)n, (TG)n, (TC)n, and (CT)n. GA was repeated 5-8 times; GT, 5 times; AG, 5-8 times; TG, 5 times; TC, 5-10 times; and CT, 11 times.

Results of genetic diversity index analyses are listed in Table 2. A total of 123 haplotypes were detected from the 124 samples; each haploid was detected in only one of the study populations.

Table 2. Genetic diversity of ITS1 among four wild Exopalaemon carinicauda populations.

\begin{tabular}{|c|c|c|c|c|c|}
\hline Population & $\mathrm{N}$ & $\mathrm{H}$ & $\mathrm{Hd}$ & $\mathrm{K}$ & $\mathrm{Pi}$ \\
\hline XingShan & 30 & 30 & 1.000 & 86.579 & 0.22844 \\
\hline NanTong & 31 & 31 & 1.000 & 92.787 & 0.30028 \\
\hline YanCheng & 31 & 31 & 1.000 & 95.501 & 0.24363 \\
\hline RiZhao & 32 & 31 & 0.998 & 95.117 & 0.25381 \\
\hline Total & 124 & 123 & 0.9999 & 84.495 & 0.28073 \\
\hline
\end{tabular}

$\mathrm{N}=$ Number of samples; $\mathrm{H}=$ number of haplotypes; $\mathrm{Hd}=$ haplotype diversity; $\mathrm{K}=$ average number of nucleotide differences; $\mathrm{Pi}$ = nucleotide diversity.

Between-population molecular variation was significant $\left(F_{\mathrm{ST}}=0.05519, \mathrm{P}<0.05\right.$; Table 3 ). The overall genetic variation was composed of $5.52 \%$ between-population, and $94.48 \%$ withinpopulation variation. Results of $F_{\mathrm{ST}}$ among the four populations are listed in Table 4 . The $F_{\mathrm{ST}}$ between population $\mathrm{R}$ and $\mathrm{N}$ was the largest (0.09324); the lowest was between population $\mathrm{X}$ and Y (0.01904).

Table 3. Analysis of molecular variance.
\begin{tabular}{lrccr}
\hline Variation source & d.f. & Sum of squares & Variance components & Percentage variation \\
\hline Among population & 3 & 1016.529 & $7.07858^{\text {va }}$ & 5.52 \\
Within population & 119 & 14421.747 & $121.19115^{\text {vb }}$ & 94.48 \\
Total variance & 122 & 15438.276 & 128.26973 & \\
\hline
\end{tabular}

Table 4. Genetic distances (upper right) and population pairwise $\mathrm{F}_{\mathrm{ST}}$ (lower left).

\begin{tabular}{lcccc}
\hline Population & $\mathrm{X}$ & $\mathrm{Y}$ & $\mathrm{R}$ & $\mathrm{N}$ \\
\hline $\mathrm{X}$ & - & 0.006 & 0.084 & 0.010 \\
$\mathrm{Y}$ & 0.01904 & - & 0.090 & 0.057 \\
$\mathrm{R}$ & 0.07503 & 0.08947 & - & 0.098 \\
$\mathrm{~N}$ & 0.02973 & 0.06770 & 0.09324 & - \\
\hline
\end{tabular}

The relevant data included ITS1 sequences from 123 haploids of E. carinicauda (obtained in this study), as well as nine ITS1 sequences of $M$. nipponense, Macrobrachium rosenbergii, and Leander modestus (obtained from GenBank), resulting in a phylogenetic tree too large to read. For this reason, 62 samples of 123 haploids were randomly selected from the four populations (15 from Y; 16 from R; 15 from N, and 16 from $X$ ) and combined with the nine ITS1 sequences from GenBank to construct the phylogenetic tree shown in Figure 2. On phylogenetic analysis, individuals of the same species, as well as species of the same genus, clustered together, indicating clear interspecific boundaries consistent with morphological classifications. 


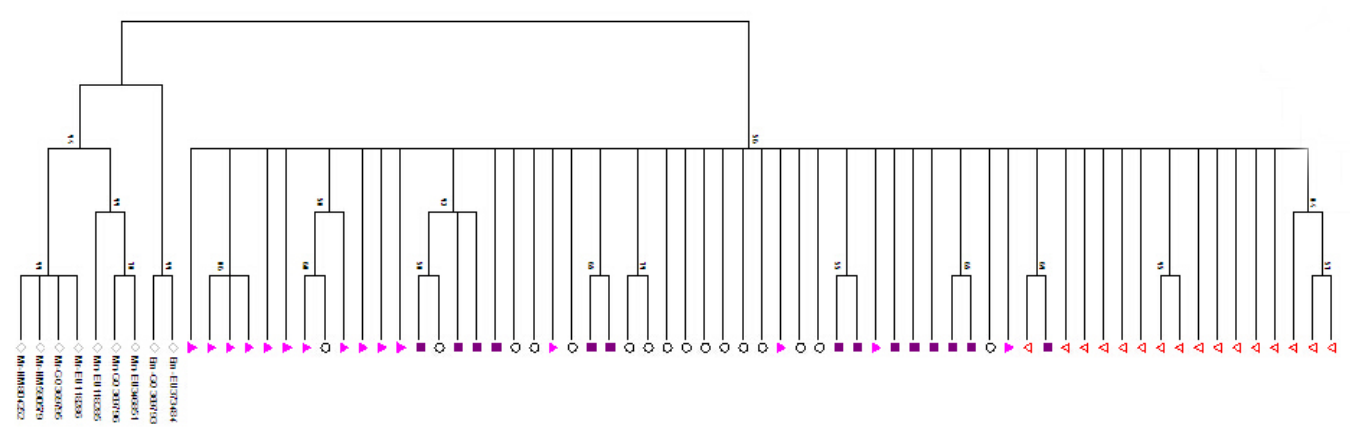

Figure 2. Phylogenetic tree of Exopalaemon sp ITS1 constructed using the neighbor-joining method. Numbers at each branch indicate the percentage of times a node was supported in 1000 bootstrap pseudoreplication. Species names and GenBank accession Nos. are as follows: Macrobrachium nipponense, GQ369796, EU346851, and EU118285; Macrobrachium rosenbergii, HM804252, HM590579, GQ369795, and EU118286; Exopalaemon modestus, GQ369793, and EU373484. Open triangles $=$ individuals from population $\mathrm{N}$; filled squares $=$ from population $\mathrm{Y}$; Filled triangles $=$ individuals from population $\mathrm{R}$; open circles = individuals from population $\mathrm{X}$.

\section{DISCUSSION}

Spacer ITS1 sequences in the RNA transcription unit of E. carinicauda were length-variable, but the length variation was not large. In combination with other Palaemonidae species sequences from GenBank, sequence lengths among species of the same genus were found to be significantly different, ranging from 345 to 384 bp for Exopalaemon, 1088 to 1485 bp for Macrobrachium. This phenomenon is generally attributed to the difference in repetitions of repeated sequences, as reported in a study on length polymorphism of ITS1 sequences in Penaeus sp (Wanna et al., 2006). Insertion or deficiency is seldom located at the repeated sequences, except for very long insertions or deficiencies in this study. Therefore, the length polymorphism of Penaeus can not be explained only by repeated sequences. This conclusion is consistent with a study on the length polymorphism of ITS1 sequences in shrimp (Wu et al., 2009). Spacer ITS1 sequences of the ribosome RNA transcription unit, located at the intron zone, have a low selection pressure and a relatively rapid evolution (Calo-Mata et al., 2009), which may explain intraspecific and interspecific length polymorphisms from one aspect (Chow et al., 2009).

A study on Drosophila showed that high GC content is a very original characteristic (Schlötterer et al., 1994). In the present study, the average GC content of the four populations was $57.9 \%$; we deduced from this observation that the Exopalaemon species are very original, with early origin and differentiation. The relatively low GC contents of Macrobrachium sp in our study may indicate late differentiation and short evolution of this particular species (Wu et al., 2009). Among the ITS1 sequences tested from 124 individuals, eight microsatellite sequences were detected, and the most-repeated sequences were (GA)n, (AG)n, (GT)n, (TG)n, (TC)n, and (CT)n. Based on the data in GenBank, 14, 9, and 4 microsatellites exist in the ITS1 sequences of $M$. nipponense, M. rosenbergii, and $L$. modestus, respectively. Microsatellite sites of (TG)n were found to be exclusive to $E$. carinicauda. Nevertheless, more data are needed to validate whether unique sites can be used as molecular markers for species identification, and whether (TG)n can be used as a molecular marker for identification of Palaemonidae.

The genetic diversity of the four populations ranked in order as: population $Y>N>R>X$; 
genetic diversity indices of population $X$ were smaller than other populations. The nucleotide diversity index and haplotype polymorphism degrees were higher compared to Penaeus merguiensis and Penaeus monodon (Wanna et al., 2006). Moreover, most individuals were found to have specific haplotypes. Results in our study, therefore, indicate that the genetic diversity indices of the four populations are relatively high.

Population genetics indicate that $F_{\mathrm{ST}}$ can indicate the between-population genetic differentiation degree, and $F_{\mathrm{ST}}=0.00-0.05$ indicates low differentiation, while $F_{\mathrm{ST}}>0.25$ indicates extremely large genetic differentiation (Relethford, 1994). In this study, between-population genetic differentiations were very weak or modest. Because population $\mathrm{R}$ is geographically remote from population $\mathrm{X}$, it could be expected that population $\mathrm{R}$ experienced the lowest gene flow with population $\mathrm{X}$, and thus has the largest genetic differentiation. However, we found a larger $F_{\mathrm{ST}}$ value between population $\mathrm{R}$ and $\mathrm{N}$ than between population $\mathrm{R}$ and $\mathrm{X}$. A possible reason for this is that population $\mathrm{R}$ lives in the relatively closed Haizhou Bay, and that the low long-distance swimming and weak diffusing abilities of $E$. carinicauda hindered gene exchange between population $\mathrm{R}$ and other populations, leading to very large genetic differentiation from the other populations. The $F_{S T}$ between population $N$ and $\mathrm{X}$, and population $\mathrm{Y}$ and $\mathrm{X}$ were very small, but are not caused by close geographical distance. These data indicate that between-population genetic difference is decided not only by betweenpopulation distance, but also by other reasons (Ortego et al., 2012). Maybe the escape of the farmed shrimp which from the same source is one of the reason for the phenomenon occurrence.

One major objective of molecular phylogeny is to construct phylogenetic trees, using base differences of DNA sequences, to clarify the relationship between different species, and between different populations of the same species (Zagoskin et al., 2014). In this study, ITS1 fragments were used to analyze the relationship between four species from two genera. Different individuals of the same species and different species of the same genus were found to cluster into one branch, consistent with morphological classifications. However, individuals among different populations did not cluster together. It may be related to the gene exchange among different populations due to various reasons. But this phenomenon, which has also been reported in a phylogenetic tree of several shrimp species (Wu et al., 2009), did not impact the clustering analysis on genus level.

Our results indicate that the genetic diversity resources of four wild $E$. carinicauda populations are well preserved. From the perspective of sustainable development and utilization of E. carinicauda, however, managers should formulate reasonable and science-based protection measures, build effective monitoring systems, and reinforce monitoring of genetic diversity in wild populations (Mandal et al., 2014). Moreover, measures should be taken to prevent blind artificial transplant, avoid deterioration of provenances and loss of fine traits caused by variety introduction, and guarantee efficient conservation and reasonable utilization of wild-type provenance resources. Due to rich nucleotide mutant sites and rapid nucleotide evolution, ITS1 is suitable for phylogenic research and genetic diversity analysis between genera, and between species of the same genus. However, there are insufficient data on Palaemonidae in GenBank, and so determination of ITS sequences in other Palaemonidae species is urgent. This task, combined with morphological classification and other molecular markers, will provide researchers with more comprehensive knowledge on the classification and phyletic evolution of Palaemonidae.

\section{ACKNOWLEDGMENTS}

Research supported by the Jiangsu Agriculture Science and Technology Innovation Fund 
[\#JASTIF)(CX(13)2040]; the Natural Science Fund for colleges and universities in Jiangsu Province (\#14KJD240001); and the Aquatic Sanxin Engineering Projects of Jiangsu Province (\#Y2014-35).

\section{REFERENCES}

Bazzicalupo AL, Bálint M and Schmitt I (2013). Comparison of ITS1 and ITS2 rDNA in 454 sequencing of hyperdiverse fungal communities. Fungal Ecol. 6: 102-109.

Calo-Mata P, Pascoal A, Fernández-No I, Böhme K, et al. (2009). Evaluation of a novel 16S rRNA/tRNAVal mitochondrial marker for the identification and phylogenetic analysis of shrimp species belonging to the superfamily Penaeoidea. Anal. Biochem. 391: 127-134.

Chow S, Ueno Y, Toyokawa M, Oohara I, et al. (2009). Preliminary analysis of length and GC content variation in the ribosomal first internal transcribed spacer (ITS1) of marine animals. Mar. Biotechnol. 11: 301-306.

Duan Y, Liu P, Li J, Li J, et al. (2013). Expression profiles of selenium dependent glutathione peroxidase and glutathione S-transferase from Exopalaemon carinicauda in response to Vibrio anguillarum and WSSV challenge. Fish Shellfish Immunol. 35: 661-670.

Estrada-Bárcenas DA, Vite-Garín T, Navarro-Barranco H, de la Torre-Arciniega R, et al. (2014). Genetic diversity of Histoplasma and Sporothrix complexes based on sequences of their ITS1-5.8S-ITS2 regions from the BOLD system. Rev. Iberoam Micol. 31: 90-94.

Fong MY, Noordin R, Lau YL, Cheong FW, et al. (2013). Comparative analysis of ITS1 nucleotide sequence reveals distinct genetic difference between Brugia malayi from Northeast Borneo and Thailand. Parasitology 140: 39-45.

Li J, Han J, Chen P, Chang Z, et al. (2012). Cloning of a heat shock protein 90 (HSP90) gene and expression analysis in the ridgetail white prawn Exopalaemon carinicauda. Fish Shellfish Immunol. 32: 1191-1197.

Mandal A, Varkey M, Sobhanan SP, Mani AK, et al. (2014). Molecular markers reveal only two mud crab species of genus Scylla (Brachyura: Portunidae) in Indiancoastal waters. Biochem. Genet. 52: 338-354.

Monard C, Gantner S and Stenlid J (2013). Utilizing ITS1 and ITS2 to study environmental fungal diversity using pyrosequencing. FEMS Microbiol. Ecol. 84: 165-175.

Ortego J, Riordan EC, Gugger PF and Sork VL (2012). Influence of environmental heterogeneity on genetic diversity and structure in an endemic southern Californian oak. Mol. Ecol. 21: 3210-3223.

Relethford JH (1994). Craniometric variation among modern human populations. Am. J. Phy. Anthropol. 95: 53-62.

Schlötterer C, Hauser MT, von Haeseler A and Tautz D (1994). Comparative evolutionary analysis of rDNA ITS regions in Drosophila. Mol. Biol. Evol. 11: 513-522.

Wang XC, Liu C, Huang L, Bengtsson-Palme J, et al. (2014). ITS: a DNA barcode better than ITS2 in eukaryotes? Mol. Ecol. Resour. 15: 573-586.

Wanna W, Chotigeat W and Phongdara A (2006). Sequence variations of the first ribosomal internal transcribed spacer of Penaeus species in Thailand. J. Exp. Mar. Biol. Ecol. 331: 64-73.

Wu Z, Feng J, Chen J, Xu S, et al. (2009). Phylogenetic analysis and species identification of popular shrimp species in southeast China using the first internally transcribed spacer of ribosomal DNA. Aquac. Res. 40: 1251-1259.

Xu W, Xie J, Shi H and Li C (2010). Hematodinium sp infection in cultured ridgetail white prawns Exopalaemon carinicauda in eastern China. Aquaculture 300: 25-31.

Zagoskin MV, Lazareva VI, Grishanin AK and Mukha DV (2014). Phylogenetic information content of Copepoda ribosomal DNA repeat units: ITS1 and ITS2 impact. Biomed. Res. Int. 2014: 926342.

Zhang C, Li F and Xiang J (2014a). Acute effects of cadmium and copper on survival, oxygen consumption, ammonia-N excretion, and metal accumulation in juvenile Exopalaemon carinicauda. Ecotoxicol. Environ. Saf. 104: 209-214.

Zhang C, Li Z, Li F and Xiang J (2014b). Effects of starvation on survival, growth and development of Exopalaemon carinicauda larvae. Aquac. Res. 1-11.

Zhao YE, Wu LP, Hu L, Xu Y, et al. (2012). Sequencing for complete rDNA sequences (18S, ITS1, 5.8S, ITS2, and 28S rDNA) of Demodex and phylogenetic analysis of Acari based on $18 \mathrm{~S}$ and 28S rDNA. Parasitol. Res. 111: 2109-2114.

Genetics and Molecular Research 14 (4): 12316-12322 (2015) 